\title{
Dulling the double-edged sword of human SEEG research
}

\author{
Guy M. McKhann II, MD \\ Department of Neurological Surgery, Columbia University Irving Medical Center, NewYork-Presbyterian Hospital, New York, \\ New York
}

I $\mathrm{N}$ patients who have focal epilepsy that is not well lateralized or localized, invasive brain recording is often recommended to pinpoint their seizure-onset zone and to determine their candidacy for potentially curative or palliative epilepsy surgery. Surgical implants for seizure recording consist of either subdural electrode arrays (with or without depth electrodes), usually implanted via a relatively large craniotomy, or stereotactically implanted depth electrodes placed through anchor bolts via a series of drill hole craniostomies (stereo-EEG [SEEG]). The potential advantages and disadvantages of these two approaches have been well described. ${ }^{6}$

Over the last half decade, the use of SEEG has rapidly spread throughout North America such that it is now the predominant invasive monitoring procedure at many comprehensive epilepsy centers. As a minimally invasive stereotactic procedure, SEEG is appealing to practitioners and patients alike because it avoids a craniotomy and has a lower morbidity rate than the alternative subdural grid and strip approach. ${ }^{6}$ SEEG electrode constructs are implanted in a 3D fashion from the outer brain surface down to deeper brain targets such as the insula, opercula, and medial hemisphere, areas traditionally difficult to access with more 2D subdural electrodes. These SEEG electrode trajectories, in addition to obtaining clinical recordings of brain activity and seizures, provide novel opportunities to study areas of the human brain not readily accessed by traditional subdural electrodes. The field of intracranial human research is thus expanding in parallel with the spread of the SEEG approach, as there are limitless questions to be posed about fundamental aspects of seizure organization and spread, systems brain physiology, human cognition and behavior, and neuromodulation, to name a few.

With new research opportunities comes the need for updated research responsibilities and safeguards, creating a double-edged sword. Every SEEG electrode placed has a small but real risk of causing morbidity and even mortality. Each clinical SEEG electrode requested by the epileptology team must be justified, based on the patient's anatomical-electrical-clinical hypothesis. Similarly, every human research project to be studied needs to utilize the anatomical locations provided by the clinically necessary electrode locations. Adding additional electrodes purely for research purposes is putting the patient at additional risk. If the planned clinical SEEG implant is altered in any way for research, these changes need to be clearly communicated to the patient preoperatively, and risk must be minimized. It is up to the epilepsy surgery field to create guidelines to make sure that risk to the patient from SEEG-based research is minimized.

SEEG electrodes are most commonly placed as close to orthogonally as possible to the skull surface to minimize the risk of the drill or electrode deflecting away from the planned trajectory. There are a finite number of electrode plans that allow spatially precise trajectories while maximizing patient safety. Expressed another way, a patient's SEEG implant strategy designed by one team at a center of excellence should appear justified and safe (and similar) to that of another experienced center's team.

In this issue of Neurosurgical Focus, Chaitanya et al. from the University of Alabama at Birmingham (UAB) present their implantation technique and complications in their first 24 patients with suspected temporal lobe epilepsy (TLE) in whom SEEG electrodes were placed to study the limbic thalamus. ${ }^{2}$ They show that the research aspect of the procedure is safe, with no symptomatic complications, while providing fundamental knowledge that will likely contribute to thalamic (possibly closed loop) stimulation in the future. In many ways, this research team provides an example of how to carry out SEEG-based human research:

1) The hypothesis is sound and likely to be scientifically fruitful. As discussed in their introduction and borne 
out by their referenced early results, the anterior and medial thalamic nuclei provide fertile ground for research into aspects of limbic epilepsy, with excellent prospects for direct patient benefit in the future. Additionally, hypothesis-based research projects (i.e., "let's see what we can find by studying this interesting location") are completely reasonable if there is a scientific rationale and if the electrodes are placed for clinical purposes. A "fishing expedition" should be avoided, and extra electrode contacts for research purposes should be justified and optimized for safety.

2) The risk to the patient is minimized. No new brain passes or atypical and possibly more dangerous trajectories are adapted for research purposes. The thalamic electrode ( 1 only) used for research purposes in each patient in the UAB series was placed into either the anterior nucleus or the medial (mediodorsal and centromedian) nuclei by extending a clinically justified opercular-insular electrode deeper into the brain to the thalamic target. It is preferable to not place additional electrode contacts solely for research purposes. However, given that anterior thalamic deep brain stimulation (DBS) is approved for the treatment of epilepsy, recording the thalamus in TLE patients is justified. In addition, the cumulative data extrapolated from DBS electrode placement without microelectrode recording, as well as data from the SEEG literature, demonstrate that the hemorrhage risk beyond the immediate cortical/subcortical electrode penetration is extremely low. $1,4,5$

3) Patients provide consent to the epilepsy neurology/research team well in advance of surgery after multiple visits. The surgeon is not part of the research consent process (see Fig. 1, Chaitanya et al. ${ }^{2}$ ). Surgeon researchers are obviously biased, while patients are known to want to please their surgeons. The surgical consent should be completely separate in time and personnel from the research consent.

4) The risk posed by the research is carefully studied. Every patient is assessed for hemorrhage or edema following electrode explantation and not just following implantation. This raises the likelihood of detecting small bleeds or areas of edema not seen on initial postimplantation localization CT scans. The UAB group found a relatively high rate $(21 \%[\mathrm{n}=5 / 24])$ of asymptomatic bleeding at or just below the electrode entry site. ${ }^{2}$ Logically, these bleeds would have been present independent of the research contacts placed into the thalamus. There were no cases of CT-detected thalamic edema or bleeding. It would be helpful if postexplantation neuropsychological testing and MRI were performed to build on the finding of no clinically significant events related to the research electrodes.
5) The learning curve is recognized. There is a learning curve to almost everything we do as neurosurgeons. For the thalamic electrodes placed by Chaitanya et al., the desired thalamic nucleus was implanted $77 \%-90 \%$ of the time. ${ }^{2}$ The imaging, planning, and surgical technique factors contributing to these inaccuracies can now be adjusted and hopefully improved.

The work by the UAB team and many other centers around the country is just in the early phase of an anticipated explosion of human intracranial research made possible by SEEG implants. The onus is on the epilepsy surgery community to responsibly carry out and monitor this work, putting patient safety first. To borrow from the safety culture of the prominent multinational engineering firm, Day \& Zimmermann, ${ }^{3}$ when it comes to surgical complications from SEEG electrode implantation, particularly when research is in play, "Why not zero?"

https://thejns.org/doi/abs/10.3171/2020.1.FOCUS2069

\section{References}

1. Cardinale F, Cossu M, Castana L, Casaceli G, Schiariti MP, Miserocchi A, et al: Stereoelectroencephalography: surgical methodology, safety, and stereotactic application accuracy in 500 procedures. Neurosurgery 72:353-366, 2013

2. Chaitanya G, Romeo AK, Ilyas A, Irannejad A, Toth E, Elsayed G, et al: Robot-assisted stereoelectroencephalography exploration of the limbic thalamus in human focal epilepsy: implantation technique and complications in the first 24 patients. Neurosurg Focus 48(4):E2, 2020

3. Day \& Zimmerman: Safety. (https://www.dayzim.com/about/ vision-values/safety) [Accessed February 7, 2020]

4. González-Martinez J, Bulacio J, Thompson S, Gale J, Smithason S, Najm I, et al: Technique, results, and complications related to robot-assisted stereoelectroencephalography. Neurosurgery 78:169-180, 2016

5. McGovern RA, Ruggieri P, Bulacio J, Najm I, Bingaman WE, Gonzalez-Martinez JA: Risk analysis of hemorrhage in stereo-electroencephalography procedures. Epilepsia 60:571-580, 2019

6. Tandon N, Tong BA, Friedman ER, Johnson JA, Von Allmen G, Thomas MS, et al: Analysis of morbidity and outcomes associated with use of subdural grids vs stereoelectroencephalography in patients with intractable epilepsy. JAMA Neurol 76:672-681, 2019

\section{Disclosures}

Dr. McKhann reports that he has a personal relationship with Medtronic as an investigator in the SLATE trial of laser ablation for temporal lobe epilepsy; he is a consultant for Koh Young Inc.

\section{Correspondence}

Guy M. McKhann II: gm317@cumc.columbia.edu.

\section{INCLUDE WHEN CITING}

DOI: $10.3171 / 2020.1 . F O C U S 2069$ 